\title{
Probabilistic traveling salesman problem: a survey
}

\author{
Abir Henchiri \\ Laboratory RIADI, University of Manouba, \\ National School of Computer Sciences (ENSI), \\ Tunisia. \\ Email: abirhenchiri89@gmail.com
}

\author{
Monia Bellalouna, Walid Khasnaji \\ Laboratory CRISTAL-GRIFT, University of Manouba, \\ National School of Computer Sciences (ENSI), \\ Tunisia. \\ Email: monia.bellalouna@gmail.com,mwkhaznaji@yahoo.fr
}

\begin{abstract}
The Probabilistic Traveling Salesman Problem (PTSP) is a variation of the classic Traveling Salesman Problem (TSP) in which only a subset of potential nodes needs to be visited on any given instance of the problem. The number of nodes to be visited each time is a random variable. The objective is to find an a priori tour which minimizes the expected length,with the strategy of visiting the present nodes in a particular instance in the same order as they appear in the a priori tour. In this paper, we survey a number of results obtained for PTSP and we present the different approaches used for solving it.
\end{abstract}

\section{INTRODUCTION}

O VER the past fifty years, the study of Combinatorial Optimization Problems (COP) has continued to grow in importance and has become one of the most active branches of discrete mathematics. This deterministic model is not adequacy with reality, where often the number of data of the studied problem is a random variable in $[[0 ; \mathrm{n}]]$.

In the late of 80 's, studies have developed on a class of combinatorial optimization problems, characterized by the fact that probabilistic elements are explicitly associated with data $\mathrm{i}, \mathrm{e}$, given an instance of the problem, only a subinstance of it will eventually be solved. This concept was called Probabilistic Combinatorial Optimization Problems (PCOPs) and was initially introduced by Jaillet [1], [2].

There are several motivations for studying the effect of including probabilistic elements in combinatorial optimization problems. The two most important motivations are, firstly, the desire to define and analyze models which are more appropriate with reality where randomness is a major source of concern. For example, for many delivery companies, only a subset of their customers requires a delivery each day. Ideally we would like to re-optimize, i .e., find an optimal TSP tour for every day. However, we may not have the resources to do this, or even if we have them it may be very time consuming to do that. It is therefore necessary to adopt a model that takes into account random phenomena. Secondly, the possibility to analyze the stability of optimal solutions to deterministic problems when the instances are disturbed by the absence of certain data.

The first problem studied in the probabilistic combinatorial optimization problems was the probabilistic traveling salesman problem [1]. Later, this approach was extended to other problems such as the probabilistic vehicle routing problem [3], the probabilistic spanning tree problem [4]. Studies on this probabilistic approach continued in many others domains such as the probabilistic maximum independent set problem [5], [6], the probabilistic longest path problem [7], the probabilistic minimum vertex covering problem [8], the probabilistic minimum coloring problem [9], the probabilistic graph-coloring in bipartite problem [10] and the probabilistic steiner tree problem [11]. The probabilistic approach has been extended on the combinatorial problems not defined on graph such probabilistic bin packing problem [12], [13] and the probabilistic scheduling problem [14].

In this paper, we interest to the well-known problem in optimization under uncertainty: the probabilistic traveling salesman problem (PTSP). The organization of the paper is as follows: section 2 presents the PCOP and its formulation and section 3 is devoted to the presentation of the research aspects of PCOP. Section 4 gives the definition and the formulation of the PTSP. A review of the main results in the literature is presented in section 5. Then, solving methods for the resolution of PTSP are presented in section 6. Finally the last section gives the concluding remarks.

\section{PRobabilistic COMBINATORIAL OPTIMIZATION PROBLEMS}

The probabilistic combinatorial optimization problems, noted PCOP are generalized versions of COP. Formally, a PCOP is defined as follows: Let $L_{n}=x_{1}, x_{2}, \ldots, x_{n}$ a finite set of data and $S$ a finite set of feasible solutions. Consider a cost function $f: S \rightarrow R$. In this model, we define on $L_{n}$ a probability law: each element $x_{i} \in L_{n}$ has a probability of $p_{i}$. The problem consists in resolving only on a subset of $L_{n}$ and then the size of the problem is a random variable. The objective is to minimize the expected objective function $E[f]$ through all parts $I$ of $P\left(L_{n}\right)$.

The most natural approach that comes in mind is to consider each potential instance as a new problem defined through the present data and to optimally solve the instance considered. This approach is called reoptimization strategy. This approach is optimal, however, it can be very much time and space consuming, in particular when the combinatorial optimization problem considered is NP-hard [15], [16], [17].

It is therefore necessary to adopt another resolution strategy, which is less costly in terms of computations. This new approach is called an a priori optimization and has been introduced in [1], [18]. It consists of determining a solution of the initial instance, where all data are present, called an $a$ 
priori solution, and applying a strategy called a modification strategy to adapt as quickly as possible the a priori solution to the subinstance that must effectively be solved.

\section{THE RESEARCH ASPECTS OF PCOP}

Since the introduction of probabilities in the formulation of COP [1], works on this subject have been considerable. Thus, we will present in this section the different research aspects addressed to these new problems.

\section{A. Study of complexity of the different strategies}

This research aspect concerns advanced study of the border between easy PCOP and PCOP hard. Most PCOP have been proven as difficult problems: like the probabilistic generalization of the shortest path which is NP-hard while the deterministic version is in $P$ and very easily solvable [18]. An interesting line of research is to try to find for a given COP case in which the probabilistic version remains easy, for example Bellalouna and al [19] found particular cases for the PTSP to be solved in an easy way.

\section{B. Asymptotic behaviour}

The asymptotic analysis of PCOPs (when the size of the problem tends to infinity) is an important and successful area of research. The results obtained in this field are very useful for several reasons, they allow to obtain approximations for problems of very large size, analyze the performance of some heuristics and finally to explore the boundary between good and bad algorithms in the probabilistic sense. An Asymptotic study was proposed for the first time by Jaillet [20] for the probabilistic traveling salesman problem. The 2-dimensional probabilistic bin packing problem was asymptotically studied by Bellalouna and al. [13].

\section{Stability Analysis}

Several research works studied the stability of COPs. For example, the studies of Hromkowic [21], Forlizzi and al. [22] etc. The objective is to study the interdependence between a solution of a given COP and the parameters that define the problem. For the probabilistic version, we call a PCOP stable if the real random variables associated to the re-optimization strategy and to the a priori strategy follow the same law.

Several studies have been devoted to the study of stable problem. We quote here Bellalouna [23], who has interested to the study of stability of the probabilistic traveling salesman problem , Boria and al [24] studied the stability of probabilistic min spanning tree in complete graph, Bouyahia and al [14] analyzed the stability of the probabilistic scheduling problems.

\section{Solving methods}

Several algorithms were implemented for the resolution of PCOP and have shown very satisfactory performance. Exact methods have been used to solve the POCP such as exact branch and bound algorithm developed by Rosenow [25] for the probabilistic traveling salesman problem. Besides that, approximate methods have received wide interests in researchers'effort to solve large scale PCOP. Among the applied approximate methods we cite the work of Bertsimas [3] who proposed and analyzed heuristics for probabilistic vehicle routing problem. In the works of Bellalouna and al ([23], [26], [12]) algorithms based on classic heuristics were proposed for the probabilistic bin packing problem. Metaheuristics were also used to solve POCP for example for probabilistic traveling salesman problem simulated annealing and tabu search were implemented [26]. A Tabu Search was implemented by Gendreau and al. [27] for the vehicle routing problem with stochastic demands and customers. Experimental studies allow to choose the best parameters for these solving methods in the probabilistic framework.

\section{DEFINITION OF THE PTSP}

The Probabilistic Traveling Salesman Problem (PTSP) is a variation of the classic Traveling Salesman Problem (TSP) and is introduced for the first time by Jaillet [1] in which only a subset of the nodes may be present in any given instance of the problem. The goal is to find an a priori tour of minimal expected length, with the strategy of visiting the present nodes in a particular instance in the same order as they appear in the a priori tour ([20], [28], [29]). The TSP can be treated as a special case of the PTSP. The main difference between PTSP and TSP is that in PTSP the probability of each node being visited is between 0 and 1 while in TSP the probability of each node being visited is 1 . In a given instance, the nodes present should be visited based on the sequence of the $a$ priori tour while the others nodes will simply be skipped.

A formulation of the problem is the following [1]: We are given an a priori PTSP tour $t$ through $n$ points of a given graph $G$. Each point $i$ is present with a probability $p_{i}$ independently of the others. Let $d(i, j)$ the distance between points $i, j$ and we assume, without loss of generality, that the a priori tour is $T=(1,2, \ldots, n, 1)$, then our problem is to find an a priori tour through all $n$ potential nodes, which minimizes the expected length of a specific a priori PTSP tour $T$, denoted $E\left[L_{T}\right]$ :

$$
\begin{aligned}
E\left[L_{T}\right]= & \sum_{i=1}^{n} \sum_{j=i+1}^{n} d_{i j} p_{i} p_{j} \prod_{k=i+1}^{j-1}\left(1-p_{k}\right) \\
& +\sum_{j=1}^{n} \sum_{i=1}^{j-1} d_{j i} p_{i} p_{j} \prod_{k=j+1}^{n} \prod_{k=1}^{i-1}\left(1-p_{k}\right)
\end{aligned}
$$

When all points $i$ have the same probability of presence $\left(p_{i}=\right.$ $p \forall i$ ), we note $q=1-p$. In this case the expected length $E\left[L_{T}\right]$ is:

$$
E\left[L_{T}\right]=p^{2} \sum_{r=0}^{n-2} q^{r} L_{T^{r}}
$$

Where $L_{T^{r}}=\sum_{i=1}^{n} d\left(i, T^{r}(i)\right) . T^{r}$ consists in jumping $r$ points from the initial tour $T$, therefore $T^{r}$ is formed by $\operatorname{pgcd}(n, r+$ 1) sub-tours. $T^{r}(i)$ the point after $i$ along the permutation $T^{r}$, thus $L_{T^{r}}$ is the length of the permutation $T^{r}$. We note that $T^{0}$ is the tour $T$ and $L_{T^{0}}$ is the length of the tour $T$. 


\section{REVIEW OF PREVIOUS RESULTS}

In [1], Jaillet derived several theoretical properties of optimal tours including the fact that such a tour may intersect itself. In [20], he bounded the relationship between optimal PTSP and TSP solutions. The analysis presented in [20] implies that under specific conditions the optimal PTSP and TSP solutions coincide. Later, others works examined some further properties of the PTSP and improved some bounds proved by Jaillet.

In this section we briefly review here the previous results.

\section{A. Properties of PTSP}

The TSP is a special case of the PTSP in which all the nodes are present ;so it is interesting to understand the relationship between the TSP and PTSP. In Jaillet's dissertation he found very special cases where TSP is stable.

Property 1. The optimum TSP tour is guaranteed to solve the PTSP optimally for problems with only 5 or fewer points (and with only 3 or fewer points when the matrix of distances between points is not symmetric).

Property 2. When the $n$ points lie at the corner points of a convex n-gon then TSP is stable.

In [20] he exhibited examples where TSP is a very bad solution for PTSP. Let us denote by $T_{P T S P}$ the optimal PTSP tour and by $T_{T S P}$ the optimal TSP tour.

Property 3. Given $D=\left(d_{i j}\right)$ the distance matrix through the $n$ points. If $D$ satisfies the triangle inequality, if the number of deterministically present points is $m$, then for $m \geq 1$ or (for $\mathrm{m}=0$ and $\mathrm{n}$ prime) :

$$
\begin{gathered}
E\left(L_{T_{P T S P}}\right) \geq p\left(1-(1-p)^{n-1}\right) L_{T_{P T S P}^{0}} \\
\frac{E\left(L_{T_{T S P}}\right)-E\left(L_{T_{P T S P}}\right)}{E\left(L_{T_{P T S P}}\right)} \leq \frac{1-p}{p} \\
\frac{L_{T_{P T S P}^{0}}-L_{T_{T S P}^{0}}^{0}}{L_{T_{T S P}}} \leq \frac{1-p}{p^{2}}
\end{gathered}
$$

These bounds are the best possible. We note that if $p$ is close to 1 , the error $\frac{1-p}{p}$ is close to zero and therefore the TSP tour is a very good approximate solution for the PTSP. If $p$ is very small the error $\frac{1-p}{p}$ tends to infinity and there is no information about the behavior of the optimal tour under TSP as solution for PTSP.

The PTSP seems much more complex than the TSP, The following results underscore the point that the probabilistic aspects of the PTSP induce some characteristics which are distinctly different from those of the TSP:

Property 4. For Euclidean plane, the optimal PTSP tour may intersect itself .
Property 5. The dynamic programming approach proposed for TSP fails to solve the PTSP.

Property 6. Given $h=(l, \ldots n)$ a path through $\mathrm{n}$ vertices, if $\mathrm{h}$ is decomposed into two paths $h_{1}=(1, . . k)$ and $h_{2}=$ $(k, \ldots, n)$ and if we note $h=h_{1} \oplus h_{2}$ then :

$$
E\left(L_{h_{1} \oplus h_{2}}\right) \leq E\left(L_{h_{1}}\right)+E\left(L_{h_{2}}\right)
$$

Functional associated to PTSP is not additive and we cannot decompose the PTSP into sub problems. The optimality principle is not verified.

Bellalouna [23], [26] found special cases where the problem is polynomial and showed that under some conditions on the distance matrix denoted $C$, TSP is stable.

She gave conditions on the constant matrices of the form $c_{i j}=a_{i}+b_{j}$ basing on a result of Berenguer [30], showing that constant matrices are the only matrices where all the permutations of vertices have the same length.

Property 7. Constant matrices are the only ones that have the same expectation for every a priori tour $T$ :

$$
E\left(L_{T_{P T S P}}\right)=p\left(1-(1-p)^{n-1}\right) L_{T_{T S P}^{0}}
$$

In this case PTSP is polynomial.

Let us call a matrix $C$ small if there exist two vertices, $a$ and $b$, such that $c_{i j}=\min \left\{a_{i}, b_{i}\right\}, i, j=1 \ldots . n . C$ is called small with distinct values where $a_{i}$ and $b_{j}$ are all distinct. In this case, let $d_{i}$ be the $i-t h$ smallest value between the $2 n$ values $a_{k}$ and $b_{j} ; D=\left\{d_{1}, \ldots . . d_{n}\right\}, \bar{D}=\left\{d_{n+1}, \ldots . . d_{2 n}\right\}$ and $d=\sum_{i=1}^{n} d_{i}$. The vertices can be partitioned into four sets: $D_{2}=\left\{i: a_{i}, b_{i} \subseteq D\right\}, D_{o}=\left\{i: a_{i}, b_{i} \subseteq \bar{D}\right\}, D_{a}=\{i:$ $\left.a_{i} \in D, b_{i} \in \bar{D}\right\}, D_{b}=\left\{i: b_{i} \in D, a_{i} \in \bar{D}\right\}$.

We remind Gabovitch's theorem [47] where he shows that for small matrices the TSP is an easy problem. In particular, he shows that the length of the optimal tour is equal to $d$ if and only if $D$ satisfies one of the following conditions:
(i) $D_{2} \neq \emptyset$,
(ii) $D=\left\{a_{l} \ldots . . a_{n}\right\}$,
(iii) $D=\left\{b_{1} \ldots . . b_{n}\right\}$.

And if the length of the optimal tour is different from $d$ then the length of the optimal tour equals $d^{\prime}=d-d_{n}+d_{n+l}$ if and only if $D^{\prime}=D \cup d_{n+l}\left\{d_{n}\right\}$ satisfies one of the following conditions:

(i') $D_{2}^{\prime} \neq \emptyset$, where $D_{2}^{\prime}$ is defined analogously to $D_{2}$,

(ii') $D^{\prime}=\left\{a_{1} \ldots . a_{n}\right\}$,

(iii') $D^{\prime}=\left\{b_{1}, \ldots . . b_{n}\right\}$.

Based on the results of Gabovitch [31], Bellalouna [23] gave conditions under which the TSP is stable.

Property 8. Let $C$ be a small matrice. Then $T_{P T S P}^{\prime}=T_{T S P}^{\prime}$ if and only if $\left[\left(d_{n}=b_{l}\right) \vee\left[\left(d_{n}=a_{l}\right) \wedge\left(d_{n-1}=b\right)\right] \wedge\left[\left(d_{n+l}=\right.\right.\right.$ $\left.\left.a_{n}\right) \vee\left[\left(d_{n+l}=b_{n}\right) \wedge\left(d_{n+2}=a_{n}\right)\right]\right]$. In this case:

$$
E\left(L_{T_{P T S P}}\right)=p\left(1-(1-p)^{n-1}\right) d^{\prime}-p^{2}\left(d_{n+1}-d_{n}\right)
$$


Property 9. Let $C$ a small with distinct values and consider the following conditions:

(cl) $\left[d_{n} \neq b_{1}\right] \wedge\left[\left(d_{n} \neq a_{1}\right) \vee\left(d_{n-1} \neq\left(b_{1}\right)\right)\right] \wedge$ $\left[\left(d_{n+1}=a_{n}\right) \vee\left(d_{n+1}=b_{n}\right) \wedge\left(d_{n+2}=a_{n}\right)\right]$

(c2) $\left[\left(d_{n}=b_{1}\right) \vee\left(d_{n}=a_{1} \wedge d_{n-1}=b_{1}\right)\right] \wedge$ $\left[\left(d_{n+1} \neq a_{n}\right) \wedge\left(d_{n+1} \neq b_{n}\right) \vee\left(d_{n+2} \neq a_{n}\right)\right]$

Then if (cl) is verified, we get:

$$
\begin{aligned}
E\left(L_{T_{P T S P}}\right)= & p\left(1-(1-p)^{n-1}\right)\left(d+a_{n}\right) \\
& -p^{2} \sum_{r=0}^{n-2} q^{r} \max \left(a_{n-r}, b_{1}\right)
\end{aligned}
$$

On the other hand, if (c2) is verified, we have:

$$
\begin{aligned}
E\left(L_{T_{P T S P}}\right)= & p\left(1-(1-p)^{n-1}\right)\left(d-b_{1}\right) \\
& +p^{2} \sum_{r=0}^{n-2} q^{r} \min \left(a_{n}, b_{1+r}\right)
\end{aligned}
$$

Bellalouna [23] was also based on the result of Lawler [31]to prove the stability of TSP. Lawler [31] showed that for triangular inequality matrix the TSP is easy then transport problem.

Property 11. Let $C$ a non negative matrix and verifies the triangular inequality, if 1 et $n$ are presents and the shortest path between 1 and $\mathrm{n}$ is $(1, \mathrm{n})$ then $T S P$ is stable.

Property 12. Let $C$ a non negative matrix and for $\mathrm{i}<$ $\mathrm{j}$ we have $c_{i, j} \leq C_{k, j} \forall k i+1 \leq k \leq j-1$ then $T S P$ is stable.

In Bellalouna'thesis [23], Christofides heuristic for TSP [31] was studied and it is proved that its approximation ratio is bounded by a constant even for the case of PTSP.

Property 13. If the TSP matrix is positive and verifies the triangular inequality and if $\mathrm{X}$ is a random variable representing the number of present vertices and verifying $\operatorname{Pr}(W \leq n-k-$ 1) $=0$ and $\operatorname{Pr}(W=n-k)>0$ then Christofides heuristic is an heuristic in the worst case for the PTSP.

$$
\frac{E\left(L_{C_{T S P}}\right)}{E\left(L_{T_{P T S P}}\right)} \leq \frac{3}{2}\left[1+\frac{k^{2}(k+1)}{n-2}\right]
$$

where $L_{C_{T S P}}$ is the tour provided by Christofides' algorithm.

Basing on research of Bellalouna relating to the small PTSP [23], [26] a study of particular cases which can be solved in an easy way was addressed in [19].

Let $T[i]$ the $i^{\text {th }}$ city of the tour $T, i \in\{1, \ldots, n\}, T[i-1]$ is the predecessor of $T[i]$ and $T[i+1]$ is the successor of $T[i]$.

Property 14. Let $C$ be a small matrix with distinct values, suppose that $D_{2}=\{1\}, D_{0}=\{n\}$ and $D_{b}=\emptyset$. Without loss of general information,let $a_{1}<a_{2}<\ldots<a_{n-1}<a_{n}$. Then: $T S P$ is stable if and only if $a_{n-1}<b_{1}$ and $a_{n}<\min \left\{b_{i}\right\}_{2 \leq i \leq n-1}$
Property 15. Let $C$ be a small matrix with distinct values, suppose that $D_{2}=\{1\}, D_{0}=\{n\}$ and $D_{b}=\{\}$. Without loss of general information,let $a_{1}<a_{2}<\ldots<a_{n-1}<a_{n}$ and $b_{1}<\ldots<b_{n-1}$ Then:

$$
T_{P T S P}=T_{T S P}^{*}
$$

\section{B. Bounds}

Bertsimas and Howell [16] improved the best upper and lower bounds for PTSP in three cases: 1) $p_{i}=p$, 2) $p_{i} \neq p_{j}$ and 3) $p_{1}=1, p_{i} \neq p_{j}$.

1) The case $p_{i}=p$. They proved that the bounds proved by Jaillet for $n$ prime (3) holds even if $n$ is not prime.

Result 2. If $T_{P T S P}$ is the optimal PTSP tour,then for $n$ not prime $(n=2 k+1)$ :

$$
E\left[L_{T_{P T S P}}\right] \geq p L_{T_{P T S P}}\left(1+(1-p)^{2 k-1}-(1-p)^{k}(2-p)\right)
$$

2) The case $p_{i} \neq p_{j}$. In the case of unequal probabilities, they obtained lower bounds for the expected length of the PTSP by using a mathematical programming rather than a combinatorial approach. They used an idea suggested by Berman and al. in [33].

Result 3. If $T_{P T S P}$ is the optimal PTSP tour, then

$$
E\left[L_{T_{P T S P}}\right] \geq z^{*}
$$

where $z^{*}$ is the optimal solution to the transportation problem.

$$
\begin{gathered}
z^{*}=\min \sum_{i, j} x_{i, j} d(i, j) \\
\text { s.t } \sum_{i} x_{i, j}=p_{j}\left(1-\prod_{k \neq i}\left(1-p_{k}\right)\right), \\
\sum_{j} x_{i, j}=p_{i}\left(1-\prod_{k \neq i}\left(1-p_{k}\right)\right), \\
x_{i, j} \geq 0
\end{gathered}
$$

For the upper bound Bertsimas and Howell [16] used the triangle inequality:

Result 4. Under the triangle inequality,

$$
E\left[L_{T_{P T S P}}\right] \leq L_{T S P}
$$

3) The case $p_{1}=1, p_{i} \neq p_{j}$. In this case they improved the bound (12) if the triangle inequality holds:

Result 5. Under the triangle inequality, any tour $T$ satisfies

$$
E\left[L_{T}\right] \leq \sum_{i=2}^{n} p i[d(i, 1)+d(1, i)]
$$

Bellalouna [23] gave also bounds for PTSP based on the work of Jaillet [1], who showed that the inequality (3) in the case where the number of points is prime. Bellalouna gave Similar results for any $n$. 
Result 6 Let $T_{P T S P}$ the optimal PTSP tour, if $n=2 k+1$ then:

$$
E\left[L_{T_{P T S P}}\right] \geq p^{2} L_{T_{P T S P}^{0}} \frac{1-(1-p)^{n-1}}{1-(1-p)^{k}}
$$

Result 7. Let $T_{P T S P}$ the optimal PTSP tour and $n=2 k>$ 6 then:

$$
E\left[L_{T_{P T S P}}\right] \geq p^{2} L_{T_{P T S P}^{0}} \frac{1-(1-p)^{n+h_{r_{0}}}}{1-(1-p)^{n}}
$$

and $\operatorname{gcd}\left(n, r_{0}+1\right)=1 \Rightarrow \exists\left(h_{n}, h_{r_{0}}\right) / n h_{n}+\left(r_{0}+1\right) h_{r_{0}}=1$.

\section{Asymptotic results}

In [2], [17] Jaillet presented an interesting analysis of the PTSP in the plane in order to find convergence results for PTSP similar to those demonstrated by Beardwood and al [34] for TSP.R

We note $x=\left\{x_{1}, x_{2}, \ldots\right\}$ a sequence of points of $R^{2}$ and $x^{n}=\left\{x_{i}, \ldots, x_{n}\right\}$. If the position of the points is random then the sequence is represented by $X=\left\{X_{1}, X_{2}, \ldots\right\}$.

Result 1. Let $X$ be a sequence of points uniformly and independently distributed within the unit square $[0,1]^{2}$ and each point has probability $\mathrm{p}$ of being present, independently of the others then there is a constant $c(p)$ as:

$$
\lim _{n \rightarrow \infty} \frac{E\left(L_{T_{P T S P}}\left(x^{n}, p\right)\right)}{\sqrt{n}}=c(p)
$$

where

$$
\beta \sqrt{p} \leq c(p) \leq \min (\beta, 0.9204 \sqrt{p})
$$

$\beta$ is the TSP constant in the theorem of Beardwood and al [34], who showed that $0.625 \leq \beta \leq 0.9204$.

\section{SOLVING METHODS}

There are several algorithms for solving PTSPs. Some papers use exact algorithms to solve PTSPs to optimality, we cite Berman and Simchi-Levi [33] who suggested a lower bound and explained how to combine this bound with a branch-and-bound algorithm to find an optimal a priori tour, Laporte and al. [35] who proposed an exact branch and cut algorithm based on an integer two-stage stochastic programming formulation to solve to optimality instances involving up to 50 vertices. Most approaches in the PTSP literature focus on heuristics that efficiently find good but not necessarily optimal solutions, these include Clarke and Wright, nearest neighbor [36], spacefilling curve, 2-OPT and 1-Shift techniques ([16], [37], [38], [39]). Recently, metaheuristics have been proposed to solve the PTSP such as simulated annealing algorithm [40], scatter search algorithm ([41], [42]), Ant Colony Optimization ([41], [43], [44], [45]) Greedy Randomized Adaptive Search Procedure (GRASP) ([46], [47]), A hybrid Honey Bees Mating Optimization (HBMO) [48], iterative local search algorithms ([49], [50]), memetic algorithms [50].

\section{CONCLUSION}

Probabilistic Combinatorial Optimization problems are very suitable and real-life problems where probabilities are associated with the data. In this paper we have interested on the PTSP the first problem studied in PCOP and we have surveyed the main results obtained on it include their combinatorial properties, bounds and asymptotic results. We have also presented the different solving methods for PTSP. A wide variety of exact and approximate algorithms have been proposed for solving it. Exact algorithms can only solve relatively small problems. As for the approximate methods, a number of heuristics and metaheuristics have proved very satisfactory for large problems. Nowadays, approximate algorithms are the main interests of many researchers who still trying to find the best algorithm which give a very good approximate solution in a proper running time.

\section{REFERENCES}

[1] P. Jaillet, "Probabilistic Traveling Salesman Problems," $\mathrm{PhD}$ thesis. MIT, Cambridge,MA, USA, 1985.

[2] P. Jaillet, "Analysis of the probabilistic traveling salesman problem in the plane," Ricerca Operativa, vol. 36, 1986.

[3] D. Bertsimas, "A Vehicule Routing Problem with Stochastic Demand," Operational Research, 1992, pp. 574-585.

[4] D. Bertsimas, "The Probabilistic Minimum Spanning Tree Problem," Networks, vol. 20, 1990, pp. 245-275.

[5] C. Murat and V. Paschos, "The probabilistic maximum independent set problem,” Proceedings ASMDA, 1995.

[6] C. Murat and V. Paschos, "The probabilistic maximum independent set problem," Theoretical Computer Science, vol. 270, 2002.

[7] C. Murat and V. Paschos, "The probabilistic longest path problem," Networks, vol. 33, 1999.

[8] C. Murat and V. Paschos, "The probabilistic minimum vertex covering problem," International Transactions on Operational Research, vol. 9, 2002 .

[9] C. Murat and V. Paschos, "The probabilistic minimum coloring problem," Proceedings WGÂŠo3, vol. 2880, pp. 346-357, 2003.

[10] N. Bourgeois, F. Della Croce, B. Escoffier, C. Murat and V. Paschos, "Probabilistic graph-coloring in bipartite and split graphs," Journal of Combinatorial Optimization, 2009.

[11] V. Paschos, O. Telelis, V. Zissimopoulos, "Probabilistic models for the Steiner Tree problem," Networks, vol. 56, pp. 39-49, 2010.

[12] M. Bellalouna, S. Souissi and B. Ycart, "Average-Case Analysis for the Probabilistic Bin Packing Problem," Trends in Mathematics, Birkh Ãd'user Verlag Basel, Switzerland, pp. 149-159, 2004.

[13] M. Bellalouna and L. Horchani, "The Two-Dimensional Probabilistic Bin-Packing Problem : An average case analysis," International Journal of mathematics and computer in simulation, pp. 42-46, 2008.

[14] Z. Bouyahia, M. Bellalouna, P. Jaillet and K. Ghedira, "A Priori Parallel Machines Scheduling," Computers Industrial Engineering, vol. 58, pp. 488-500, 2010.

[15] D.J. Bertsimas, P. Jaillet, A. Odoni, "A priori optimization," Operations Research, vol. 38(6),pp. 1019-1033, 1990. 
[16] D.J. Bertsimas, Louis H . Howell "Further results on the probabilistic traveling salesman problem," European Journal of Operational Research, vol. 65, pp. 68-95, 1993.

[17] P. Jaillet, "Analysis of combinatorial optimization problems in the euclidean spaces,” Mathematics of Operation Research, vol. 18(1), 1993.

[18] D.J. Bertsimas, "Probabilistic combinatorial optimization problems, “ $\mathrm{PhD}$ thesis, MIT, Cambridge, MA, 1988.

[19] M. Bellalouna,V.Th.Paschos and W.Khaznaji, "Well solved cases of probabilistic traveling salesman problem," 42emes Journees de Statistique, 2010.

[20] P. Jaillet, "On some probabilistic combinatorial optimization problems defined on graphs," Flow Control of Congested Network, vol. 38, pp. 255-267, 1987.

[21] J. Hromkovic, "Stability of approximation algorithms for hard optimization problems," PSOFSEM'99: Theory and Practice of Informatics, vol. 1725, pp. 29-47, 1999.

[22] L. Forlizzi, J. Hromkovic, G.Proietti, and S. Seibert, "On the stability of approximation for hamiltonian path problem," Proc. Theory and Practice of Comp. Sci., vol.3381, pp. 147-156, 2005.

[23] M. Bellalouna, "Problèmes d'optimisation combinatoires probabilistes," $\mathrm{PhD}$ thesis, CERMA, Ecole Nationale des Ponts et Chausstes, Paris, 1993.

[24] N. Boria, C. Murat, V. Paschos, "The small traveling salesman problem," Journal of Mathematical Modelling and Algorithms, vol. 11, pp. 45-76, 2012.

[25] S. Rosenow, "Comparison of an Exact Branch-and-Bound and an Approximative Evolutionary Algorithm for the Probabilistic Traveling Salesman Problem," Operations Research Proceedings, pp. 168-174, 1998.

[26] M. Bellalouna, C. Murat, and V.Th. Paschos, "Probabilistic combinatorial optimization problems on graphs : A new domain in operational research," European Journal of Operational Research, vol. 87(3), pp. 693-706, 1995.

[27] M. Gendreau,G. Laporte, R. Séguin, "A Tabu Search Heuristic for the Vehicle Routing Problem with Stochastic Demands and Customers,' Operations Research, vol. 44, pp. 469-477, 1996.

[28] P. Jaillet, "A priori solution of a Travelling Salesman Problem in which a random subset of the customers are visited," Operations research, vol. 36, pp. 929-936, 1988.

[29] P. Jaillet, "Rates of convergence of quasi additive smooth Euclidean funcionals and application to combinatorial optimization problems," Mathematics of Operations Resaerch, vol. 17, pp. 965-980, 1992.

[30] X. Berenguer, "A characterisation of linear admissible transformation for the m-traveling salesmen problem," European J.Oper.Res., vol. 3, pp. 232-249, 1979.

[31] Gabovitch, E.Y., "The small traveling salesman problem," Trudy Vychisl. Tsentra Tratu., Gos. Univ, vol. 19, pp. 27-51, 1970.

[32] E.L. Lawler, J.K. Lenstra, A.H.G. Rinnooy Kan, and D.B.Shmoys, "The Traveling Salesman Problem," John Wiley and Sons, New york, 1985.

[33] O. Berman and D. Simchi-Levi, "Finding the optimal a priori tour and location of a traveling salesman with nonhomogeneous customers," Transportation Science, vol. 22(2),pp. 148-154, 1988.

[34] J. Beardwood, J. Halton, and J. Hammersley, "The shortest path through many points," Proc. Camb. Phil Soc, vol. 55, pp. 299-327, 1959.
[35] G. Laporte, F. Louveaux, and H. Mercure, "A Priori Optimization of the Probabilistic Traveling Salesman Problem," Operations Research, vol. 42(3),pp. 543-549, 1994.

[36] F. A. Rossi and I. Gavioli, "Aspects of heuristic methods in the probabilistic traveling salesman problem," Advanced School on Statistics in Combinatorial Optimization, pp. 214-227, 1987.

[37] D.J. Bertsimas, P. Chervi, M. Peterson,"Computational approaches to stochastic vehicle routing problems," Transportation Science, vol. 29(4),pp. 342-352, 1995.

[38] L. Bianchi, J. Knowles, N. Bowler, "Local search for the probabilistic traveling salesman problem : Correction to the 2-p-opt and 1- shift algorithms" European Journal of Operational Research, vol. 162(1), pp. 206-219, 2005.

[39] L. Bianchi, A. M. Campbell, "Extension of the 2-p-opt and 1-shift algorithms to the heterogeneous probabilistic traveling salesman problem," European Journal of Operational Research vol. 176, pp. 131144, 2007.

[40] N. E. Bowler, T. M. A. Fink, and R. C. Ball, "Characterization of the probabilistic traveling salesman problem," Physical Review, E 68, 036703,2003

[41] Y.-H. Liu, "A hybrid scatter search for the probabilistic traveling salesman problem,” Comput. Oper. Res, vol. 34, pp. 2949-2963, 2007.

[42] Y.-H. Liu, "Diversified local search strategy under scatter search framework for the probabilistic traveling salesman problem," Eur. J. Oper. Res., vol. 191,pp. 332-346, 2008.

[43] L. Bianchi, L. M. Gambardella, and M. Dorigo, "An ant colony optimization approach to the probabilistic traveling salesman problem," 7th International Conference on Parallel Problem Solving from NatureI, vol. 2439, pp 883-892,2002.

[44] L. Bianchi, L. M. Gambardella, and M. Dorigo, "Solving the homogeneous probabilistic traveling salesman problem by the ACO metaheuristic," 3rd International Workshop on Ant Algorithms, vol. 2463, pp 176-187,2002.

[45] L. Bianchi, "Ant colony optimization and local search for the probabilistic traveling salesman problem : a case study in stochastic combinatorial optimization," Ph.D. Thesis, Univ. Libre de Bruxelles, 2006.

[46] Y. Marinakis, M. Marinaki, "A hybrid multi-swarm particle optimization algorithm for the probabilistic traveling salesman problem," Comput. Oper. Res., vol. 37, pp. 432-442, 2010.

[47] Y. Marinakis, A. Migdalas, P.M. Pardalos, "Expanding neighborhood search - GRASP for the probabilistic traveling salesman problem,' Optim. Lett, vol. 2, pp. 351-361, 2008.

[48] Y. Marinakis, M. Marinaki, "A hybrid honey bees mating optimization algorithm for the probabilistic traveling salesman problem,' BIBLIOGRAPHIE 24 Proc.IEEE Congr. Evol. Comput., CEC '09, pp. 1762-1769, 2010.

[49] P. Balaprakash, M. Birattari, T. StÃijtzle, M. Dorigo, "Estimationbased local search for stochastic combinatorial optimization using delta evaluations : a case study on the probabilistic traveling salesman problem," INFORMS J. Comput, vol. 20, pp. 644-658, 2008.

[50] P. Balaprakash, M. Birattari, T. Stützle, M. Dorigo, "Adaptive sample size and importance sampling in estimation-based local search for the probabilistic traveling salesman problem," European J. Oper.Res., vol. 199, pp.98-110, 2009 